\title{
1 Gene content of the fish-hunting cone snail Conus consors
}

2 Reidar Andreson ${ }^{1,2,3}$, Märt Roosaare ${ }^{1}$, Lauris Kaplinski ${ }^{1,2}$, Silja Laht ${ }^{1,2}$, Triinu Kõressaar ${ }^{1}$,

3 Maarja Lepamets ${ }^{1}$, Age Brauer ${ }^{1,2}$, Viktorija Kukuškina ${ }^{2}$ and Maido Remm ${ }^{1,2}$

4

5 1) Department of Bioinformatics, University of Tartu, Riia 23, EE-51010 Tartu, Estonia

6 2) Estonian Biocentre, Riia 23, EE-51010 Tartu, Estonia

7 3) Institute of Genomics, Riia 23, EE-51010 Tartu, Estonia

9 Corresponding author: Reidar Andreson

10 Email: reidar.andreson@ut.ee

11 Mailing address: 23 Riia str., EE-51010 Tartu, Estonia

12 Tel: $+372-7374047$

13 


\section{Abstract}

Background. Conus consors is a fish-hunting cone snail that lives in the tropical waters of the

17 Indo-Pacific region. Cone snails have attracted scientific interest for the amazing potency of their venom, which consists of a complex mixture of small proteins known as conopeptides, many of which act as ion channel and receptor modulators with high selectivity.

Results. We have analysed publicly available transcriptomic sequences from 8 tissues of Conus consors and complemented the transcriptome data with the data from genomic DNA reads. We identified 17,715 full-length protein sequences from the transcriptome. In addition, we predicted

23168 full-length or partial conopeptide sequences and characterized gene structures of several 24 conopeptide superfamilies.

\section{Introduction}

27 Conus consors is a marine gastropod of the species-rich and highly diverse Mollusca phylum and we present the first extensive study of this organism from a genomic point of view. The first few genomes from this phylum (California sea hare, pearl oyster, Pacific oyster, owl limpet, octopus, and a freshwater snail) have only recently been sequenced (Takeuchi et al., 2012; Zhang et al.,

31 2012; Simakov et al., 2013; Albertin et al., 2015; Adema et al., 2017) The phylogenetic position

32 of C. consors is provided in Figure 1, which was constructed with particular reference to the

33 other mollusc species for which genomic data are available.

34 C. consors is a member of the Conoidea superfamily that consists of more than 700 species

35 worldwide (Puillandre et al., 2014; Lavergne et al., 2015; Gao et al., 2017). C. consors lives in

36 the tropical waters of the Indo-Pacific, inhabits sub-tidal coastlines, but is also found at depths of 
37 up to 200 meters, where it buries itself under sand and silt for shelter

38 (http://biology.burke.washington.edu/conus/).

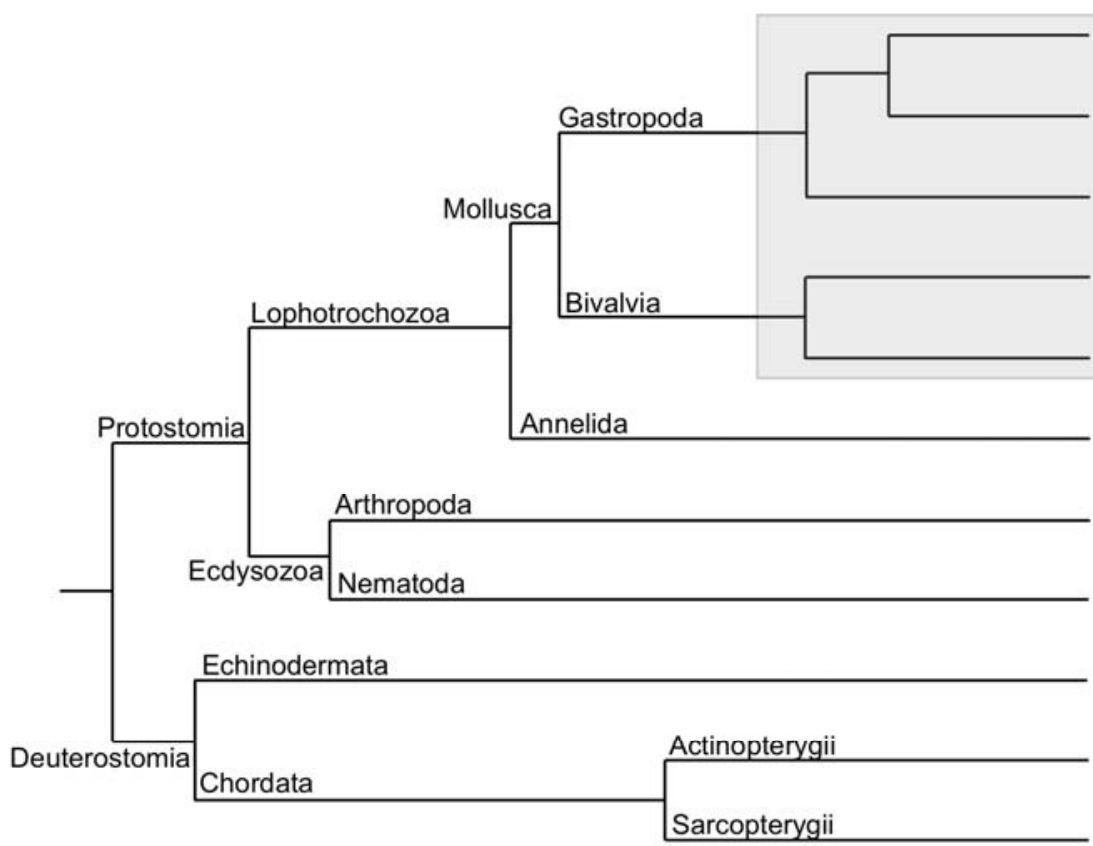

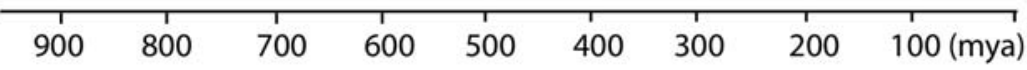

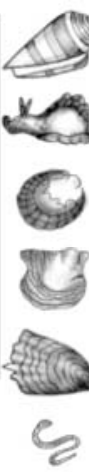

Conus consors

Aplysia californica

Lottia gigantea

Pinctada fucata

Crassostrea gigas

Capitella capitata

Drosophila melanogaster

Caenorhabditis elegans

Strongylocentrotus purpuratus

Danio rerio

Homo sapiens

\section{Figure 1. Phylogenetic position of $C$. consors in relation to some model organisms with}

42 sequenced genomes. The divergence times were obtained from the "Timetree of life" project

43 (Hedges et al., 2015). Phylogenetic relationships within the Mollusca phylum are based on

44 (Smith et al., 2011b) and (Kocot et al., 2011). The nodes included in the grey box are not time-

45 scaled.

47 The cone snails have attracted scientific interest because of their pharmacologically active

48 venom, which may provide leads in the search for novel drugs. The venom is a complex mixture 
49 of small peptides, termed conopeptides, that primarily act as ion channel modulators (Han et al.,

50 2008; Favreau \& Stöcklin, 2009; Lewis et al., 2012; Neves et al., 2015; Mir et al., 2016; Liu et

51 al., 2018). When $C$. consors injects a fish with its venom, the fish is paralyzed within a few

52 seconds and secured via a harpoon-like device. This "hook and line" strategy (Olivera, 1997) is

53 unique to cone snails and makes up for their inability to chase prey.

54 Previous peptidomic and proteomic studies have revealed that the venom of cone snails is a

55 complex mixture of several hundred peptides that shows both inter- and intra-species specific

56 variability (Biass et al., 2009; Dutertre et al., 2010, 2013, 2014; Abdel-Rahman et al., 2011; Fu et

57 al., 2018). Some variations in venom properties are linked to predation or defence stimuli

58 (Dutertre et al., 2014).

59 To gain insight into the complexity of $C$. consors, we analysed transcriptome and genome

60 sequences with the focus on gene content.

\section{Materials and Methods}

\section{Transcriptome assembly}

64 For assembly, we used publicly available sequencing reads generated by the CONCO consortium

65 (Project \#PRJNA271554 at NCBI SRA database). The transcriptome assembly included three

66 steps: pre-processing of raw reads, separate assembly of tissue-specific transcriptomes from eight

67 different tissues (venom duct, salivary gland, nerve ganglion, osphradium, mantle, foot,

68 proboscis, and venom bulb) and combining transcriptomes into one non-redundant transcriptome 69 set. 
70 For pre-processing we trimmed the low quality 3'-ends of Illumina paired-end reads with the

71 FASTQ Quality Trimmer from the FASTX Toolkit package version 0.0.13

72 (http://hannonlab.cshl.edu/fastx_toolkit/) using the quality cut-off (“"-t”) at 30 and set the

73 minimum length of the reads (“-1") at $50 \mathrm{bp}$. We cleaned the reads with DeconSeq 0.4.1

74 (Schmieder \& Edwards, 2011) and almost 850 million reads remained (in total 800 Gbps).

75 For assembly of the transcriptome, we used the Trinity assembler (version 2012-06-08)

76 (Grabherr et al., 2011) to create de novo transcripts for each sample with a minimum assembled

77 contig length (“--min_contig_length”) set to 201 nucleotides.

78 Finally, in order to obtain a non-redundant set of sequences, we clustered the transcripts with

79 CD-HIT-EST (Li \& Godzik, 2006) using a sequence identity threshold (“-c”) of 0.98. The

80 clustered transcriptome set is called the TRINITY transcriptome.

Genome assembly

83 We have used publicly available sequencing reads generated by the CONCO consortium using a

84 Roche 454 Genome Sequencer and an Illumina/Solexa GAII (Project \#PRJNA267645 at NCBI

85 SRA database). The average lengths of Roche 454 and Illumina reads were 354 bp and 104 bp,

86 respectively. Four different types of data were used for the genome assembly: Roche 454

87 shotgun-sequenced reads, artificial 454 reads from an Illumina preliminary assembly with

88 SOAPdenovo, six libraries of Illumina paired-end reads (300 bp and $600 \mathrm{bp}$ insert sizes), and

89 three libraries of Illumina mate pair reads (1.2 kbp, $3 \mathrm{kbp}$, and $7 \mathrm{kbp}$ insert sizes). Detailed

90 specifications for these libraries are provided in Supplemental Article S1. 
91 During pre-processing, low quality 3' ends of Roche 454 and Illumina reads were trimmed with

92 the FASTQ Quality Trimmer. A quality cut-off ("-t") was set to 30 and the minimum length of

93 the reads (“-1") was set to $50 \mathrm{bp}$. Consequently, reads were cleaned of human and bacterial

94 contamination with DeconSeq 0.4.1. Identity ("-i") and coverage ("-c") cut-offs of 90\% were

95 used when scanning reads against human genome NCBI GRCh37 patch release 8 and 2,370

96 different bacterial strains. For the third step, SeqClean (version 2011-02-22)

97 (https://sourceforge.net/projects/seqclean/) was used to remove any vector contaminations,

98 linkers or adapter sequences. Tool was executed with default parameters excepting a minimum

99 length of valid reads ("-1 50"), trimming of polyA/T tails, and low-complexity screening was

100 disabled ("-A -L”). Reads were scanned against UniVec database build 7.0

101 (http://www.ncbi.nlm.nih.gov/tools/vecscreen/univec/) to remove any vector sequences.

102

103

104

105

106

107

108

109

110

111 112 final assembly.

Assembly included two distinct steps. At first, SOAPdenovo 2.04 (Luo et al., 2012) was used to create the initial genome assembly with Illumina paired-end/mate-pair reads. The goal was to create 454 "pseudo-reads" from the Illumina assembly as additional input data for Newbler. SOAPdenovo was applied with a k-mer word size of 37. The SOAPdenovo assembly generated many scaffolds that contained unresolved gaps (strings of "N"s). These scaffolds were split into $300 \mathrm{bp}$ long sub-sequences with 200 bp overlaps to eliminate incorrect estimation of gap sizes using EMBOSS splitter (Rice, Longden \& Bleasby, 2000). As a second step, Newbler 2.7 (https://sequencing.roche.com/) was run with the parameters "-large -rip -mi 98 -ml 100" to assemble all three types of reads - 454 (maximum read length 1,892 bp), "pseudo" 454 (300 bp) and Illumina (145 bp) - into one unique dataset. Contigs longer than 200 bp were reported in 


\section{Discovery of full-length genes from the transcriptome}

115 We compiled a list of full-length genes from the TRINITY transcriptome using the following

116 criteria:

117 1. We selected transcripts that exhibit at least $95 \%$ of their length matched to the genome using a

118 BLASTN (version 2.2.22) (Altschul et al., 1997) alignment search. We performed unique

119 mapping by first finding pairwise alignments between a transcript and a genomic region where

120 the given alignment had the highest homology bitscore for both the transcript and genomic

121 regions (seeds). For each seed we added the alignments for which the same transcript had highest

122 alignment bitscore with the given genomic regions.

123 2. We annotated these transcripts using a BLASTX homology search against the UniRef100

124 database (Nov. 15, 2013) (Suzek et al., 2007). When homology to a given protein reached at

125 least $75 \%$, we annotated the transcript with its putative corresponding protein. In cases where

126 there were multiple candidate proteins, we chose the one with highest cumulative alignment

127 bitscore.

128 3. The cumulative bitscore of all transcript alignments with a given protein had to be greater than 129 or equal to 100 bits.

130 4. All partial transcript homologies with a given protein had to be in the same translational 131 frame.

132 5. The Open Reading Frame (ORF) had to be in one single translational frame, i.e. both the start 133 and stop codons were present in the same frame. 
134 6. The ORF start codon had to be located no more than 10 amino acids after the start of the first

135 alignment and the stop codon not more than 10 amino acids before the end of the last alignment.

136 In cases where all of these criteria were met, we assigned the protein from the UniRef100

137 database as the annotation of a given transcript and generated the predicted protein sequence

138 from the ORF.

\section{Annotation of conopeptides}

141 We used four approaches to annotate conopeptide sequences from the assembled genome: 1) a

142 BLAST search against the UniProtKB/Swiss-Prot database (release 2012_10) (The UniProt

143 Consortium, 2015); 2) a HMM search using software HMMER 3.0 (http://hmmer.org/) (Eddy,

144 2011) against conopeptide HMM profiles (Laht et al., 2012); 3) a BLAST search against peptide

145 sequences from $C$. consors venom proteomic data (Violette et al., 2012); and 4) a BLAST search

146 against conopeptide sequences predicted from the transcriptome data of $C$. consors. In all four

147 cases we applied an E-value cut-off of $10^{-5}$. We ran the HMMER and BLAST searches with

148 default parameter values, except that we turned off the BLAST filtering option (-F F). We

149 discarded matches that covered less than $50 \%$ of the length of their respective HMM profiles.

150 We manually assessed the alignments and domain boundaries for all predictions.

152 Data availability

153 Draft genome assembly of the cone snail can be retrieved from the GenBank database with

154 following assembly ID: GCA_004193615. Gene and protein sequences predicted from

155 transcriptome are included in Supplemental Data S4 (in FASTA format). 
157 Results and Discussion

158 Transcriptome and genome assembly

159 Transcriptome assemblies were created with Trinity software using read libraries from eight

160 different tissues (venom duct, salivary gland, nerve ganglion, osphradium, mantle, foot,

161 proboscis, and venom bulb). The total number of transcripts (including isoforms) was 1,535,709

162 and ranged from 85,807 ("Foot" sample) to 240,307 ("Mantle" sample) and contained around

$1631,062 \mathrm{Gbp}$ of sequence. The average length of the resulting transcripts for all samples was 692

$164 \mathrm{bp}, \mathrm{N} 50=2,452 \mathrm{bp}$, and the longest sequence was $29,867 \mathrm{bp}$. After clustering the results from

165 eight samples with CD-HIT-EST, the final dataset contains 587,852 transcripts ( 324 Gbp in total). The transcriptome data was used to compile a full-length gene list and to predict

167 conopeptide genes.

For genome assembly we used a strategy similar to the one employed to assemble the genome of the fire ant Solenopsis invicta (Wurm et al., 2011). Briefly, this strategy consisted of two major steps: (a) assembly of Illumina reads (9 libraries, overall $51 \mathrm{Gbp}$ of raw data) into larger contigs

171 using SOAPdenovo software and (b) combining the resulting Illumina contigs and original

172 paired-end reads from the Illumina and unpaired reads from Roche 454 libraries (1 fragment

173 library, overall $6 \mathrm{Gbp}$ of raw data) into a final assembly using the software Newbler

174 (Supplemental Article S1 Figure 1). The assembly of Illumina reads into longer artificial reads

175 was required because Newbler is not optimized to work with short Illumina reads. In step (b), the 176 original Illumina reads were also included to provide additional information about the distance 177 between paired reads. 
The final assembly of Conus consors genomic reads resulted in a 2,049 Mbp sequence consisting of 2,688,687 scaffolds and contigs with an N50 size of 1,128 bp. Newbler software is able to estimate the size of the entire genome based on k-mer frequency distribution. C. consors genome was estimated to be $3.025 \mathrm{Gbp}$, which is within the range of other cone snail genomes (http://genomesize.com/). The genomic DNA resulting from this assembly is fragmentary; nevertheless, the protein-coding exons are generally contiguous. Therefore, we were able to use it as an additional source of information in gene prediction process and for characterization of conopeptide gene structures.

The genome of $C$. consors is rich in repeats. Approximately $49 \%$ of the genome sequence contains repeated sequences, half of which are low-complexity (mononucleotide, dinucleotide, trinucleotide and tetranucleotide) repeat elements. Detailed analysis of repeat elements present in the genome is shown in Supplemental Article S1.

\section{Coverage of core genes in transcriptome and genome}

To evaluate the completeness of our transcriptome and genome assemblies we calculated the length coverage of core genes from the Core Eukaryotic Genes Mapping Approach (CEGMA) dataset (Parra, Bradnam \& Korf, 2007; Parra et al., 2009). This dataset consists of 458 core proteins that are universally present in 6 eukaryotic species: Homo sapiens, Drosophila melanogaster, Arabidopsis thaliana, Caenorhabditis elegans, Saccharomyces cerevisiae and Schizosaccharomyces pombe. A similar method has previously been used to evaluate the quality of two different ant genome assemblies (Smith et al. 2011; Wurm et al. 2011). Coverage (fraction of amino acids detected by TBLASTN search using core protein dataset as a query) of 
200

201

202

203

204

205

206

core genes in our transcriptome and genome data is shown in Figure 2. The median coverage of core genes is $99.7 \%$ for transcriptome and $93.4 \%$ for the genome. Similar genome coverage was observed for other mollusc genomes (Supplemental Article S1). One has to take into account that TBLASTN is somewhat limited in finding short exons in genome, thus the coverage of core genes measured from genome will always be lower than coverage in transcriptome. An illustration of core gene alignment from C. consors genome is shown in Figure 3.

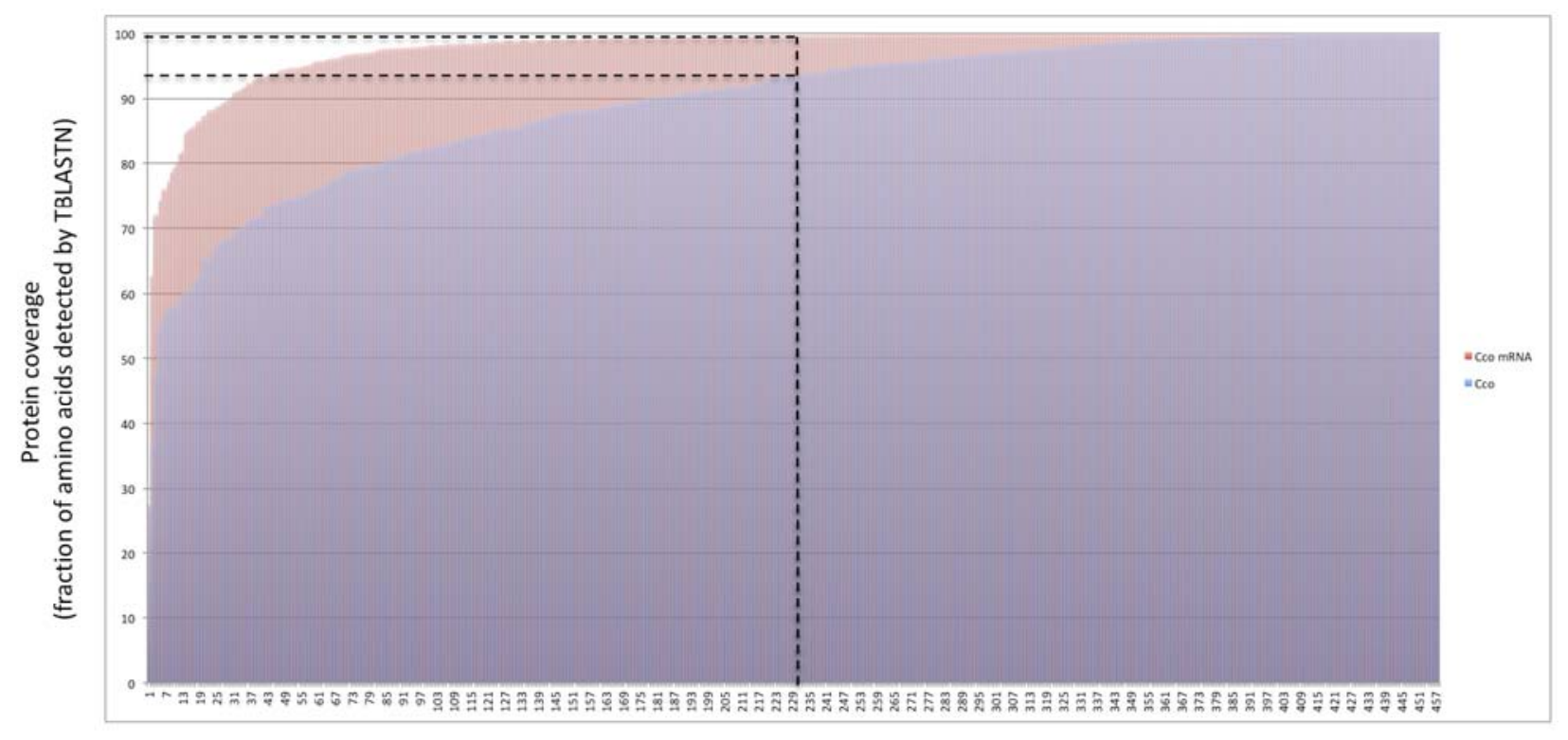

208 Figure 2. Coverage of 458 core proteins from the CEGMA dataset in C. consors transcriptome and genome. Coverage is defined as fraction of amino acids detected by TBLASTN search using core protein dataset as a query.

\section{Gene content of $\boldsymbol{C}$. consors}

212 We predicted full-length protein sequences from the transcriptome data using a reciprocal

213 homology search between the transcriptome and the UniRef100 protein database. The genome 
214 sequence was used to confirm the existence of genes predicted from transcriptome. We consider

215 the resulting 17,715 full-length proteins to be a reliable prediction of protein-coding sequences of

216 C. consors. The collection of mRNAs and translated protein sequences in FASTA format is

217 available in Supplemental Data S4. It has to be kept in mind that the actual number of protein-

218 coding genes is somewhat larger due to the fact that transcriptome analysis cannot reveal genes

219 that are expressed at low levels, in other tissues or just temporarily.

>HS4507761_KOG0003 (UBA52, ubiquitin A-52 residue ribosomal protein fusion product 1) Coverage: $128 / 128$ (100\%)

MQIFVKTLTGKTITLEVEPSDTIENVKAKIQDKEGIPPDQQRLIFAGKQLEDGRTLSDYNIQKESTLHLVLRLRGGIIEPSLRQLAQKYNCDKMICRKCYARLHPRAVNCRKKKCGHTNNLRPKKKVK

1 MQIFVKTLTGKTITLEVEPSDTIENVKAKIQDKEG 35

MQIFVKTLTGKTITLEVEPSDTIENVKAKIQDKEG

11 MQIFVKTLTGKTITLEVEPSDTIENVKAKIQDKEG 115

$\begin{array}{ccll}\text { Query } & 35 & \text { GIPPDQQRLIFAGKQLEDGRTLSDYNIQK } & \text { GTLIL } \\ \text { Sbjct } & 603 & \text { GIPPDQQRLIFAGKQLEDGRTLSDYNIQK } & 69 \\ \text { GIPPDQRLIFAGKQLEDGRTLSDYNIQK } & 707\end{array}$

Query 64 ESTLHLVLRLRGGIIEPSLRQLAQKYNCDKMICRK 98

ESTLHLVLRLRGGIIEPSLR LA KYNCDKMICRK

Sbjct 312 ESTLHLVLRLRGGIIEPSLRILASKYNCDKMICRK 416

Query 98 CYARLHPRAVNCRKKKCGHTNNLRPKKKVK 128

sbjet 970 CYARLHPRA NCRK+KCGHT+N+RPKKK+K

\begin{tabular}{|c|c|}
\hline $\begin{array}{c}\text { contig912458 } \\
\text { length }=781 \\
\text { contig912458 } \\
\text { length }=781\end{array}$ \\
\hline
\end{tabular}

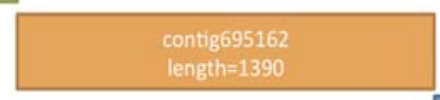

222 Figure 3. Example of gene content in the genome. TBLASTN against the genome using

223 CEGMA (core protein set present in all eukaryotes) protein Hs4507761 as a query. Red and

224 green text denote location of alternating exons in the human gene UBA52. Red orange and blue

225 boxes are matching regions from contigs or scaffolds of the $C$. consors genome. Alignment

226 between Hs4507761 and translated genomic DNA is shown in the middle. 
228 We analysed the number of tRNA, rRNA and of other non-protein-coding genes using

229 tRNAScan-SE (Lowe \& Eddy, 1997) and Infernal software (Nawrocki, Kolbe \& Eddy, 2009).

230 We detected a total of 761 different tRNA genes in the C. consors genome, 2500 miRNA genes

231 and many other types of RNA genes. Detailed analysis of RNA genes present in the genome is

232 shown in Supplemental Article S1 and full list of detected RNA genes is shown in Supplemental

233 Article S1 Table 2.

235 Conopeptide sequences

236 To identify conopeptide sequences in the transcriptome and genome of C. consors, we used

237 several sources of data with previously known conopeptide sequences or hidden Markov model

238 (HMM) profiles. Conopeptide sequences available in the UniProtKB/Swiss-Prot database (975

239 peptides from more than 30 different superfamilies), 64 conopeptide hidden Markov model

240 (HMM) profiles from 20 different superfamilies (Laht et al., 2012), 126 peptide sequences from

241 the C. consors proteome sequencing (Violette et al., 2012), and conopeptide precursor sequences

242 predicted from the transcriptome data (135 distinct precursor sequences from 23 different

243 superfamilies) were used. In addition to main transcriptome data we also used another dataset

244 (CC8 transcriptome), sequenced earlier. This additional transcriptome data originated from two

245 ESTs libraries constructed from venom duct and salivary gland tissues. The procedure for

246 obtaining CC8 transcriptome sequences is described in (Terrat et al., 2012). The genome

247 sequence was also checked for potential conopeptide genes in hope that it complements

248 transcriptome-based data. 
249 To estimate the overall number of conopeptides encoded by $C$. consors, we aligned predicted

250 protein sequences obtained from the genome, transcriptome, and proteome into multiple

251 alignments (Supplemental Data S3.). Sequences from different datasets exhibit clear clusters

252 with slight variations between individual sequences. Closely related sequences were merged into

253 clusters if the difference between sequences did not exceed 4 amino acids and the overall number

254 of sequence clusters was counted. Example of multiple alignment of sequences from the O1-

255 superfamily is shown in Figure 4. This way we estimated that $C$. consors could have at least 168

256 conopeptides: 27 with previously known sequence and 141 novel sequences. In addition, we list

25746 dubious sequences, which were only detected in the genome and did not have any closely

258 related sequence in databases. These might be products of pseudogenes, products of wrongly

259 predicted genes or peptides with other functions. However, it is not excluded that some of these

260 "dubious" clusters might represent novel conopeptides. The superfamilies M, O1, and A

261 comprise about $42 \%$ of all identified conopeptides in the $C$. consors (Table 1), which is in

262 concordance with previously published data (Puillandre et al., 2012). 
bioRxiv preprint doi: https://doi org/10.1101/590695; this version posted March 28, 2019. The copyright holder for this preprint (which was not certified by peer review) is the author/funder, who has granted bioRxiv a license to display the preprint in perpetuity. It is made available under aCC-BY-NC-ND 4.0 International license.

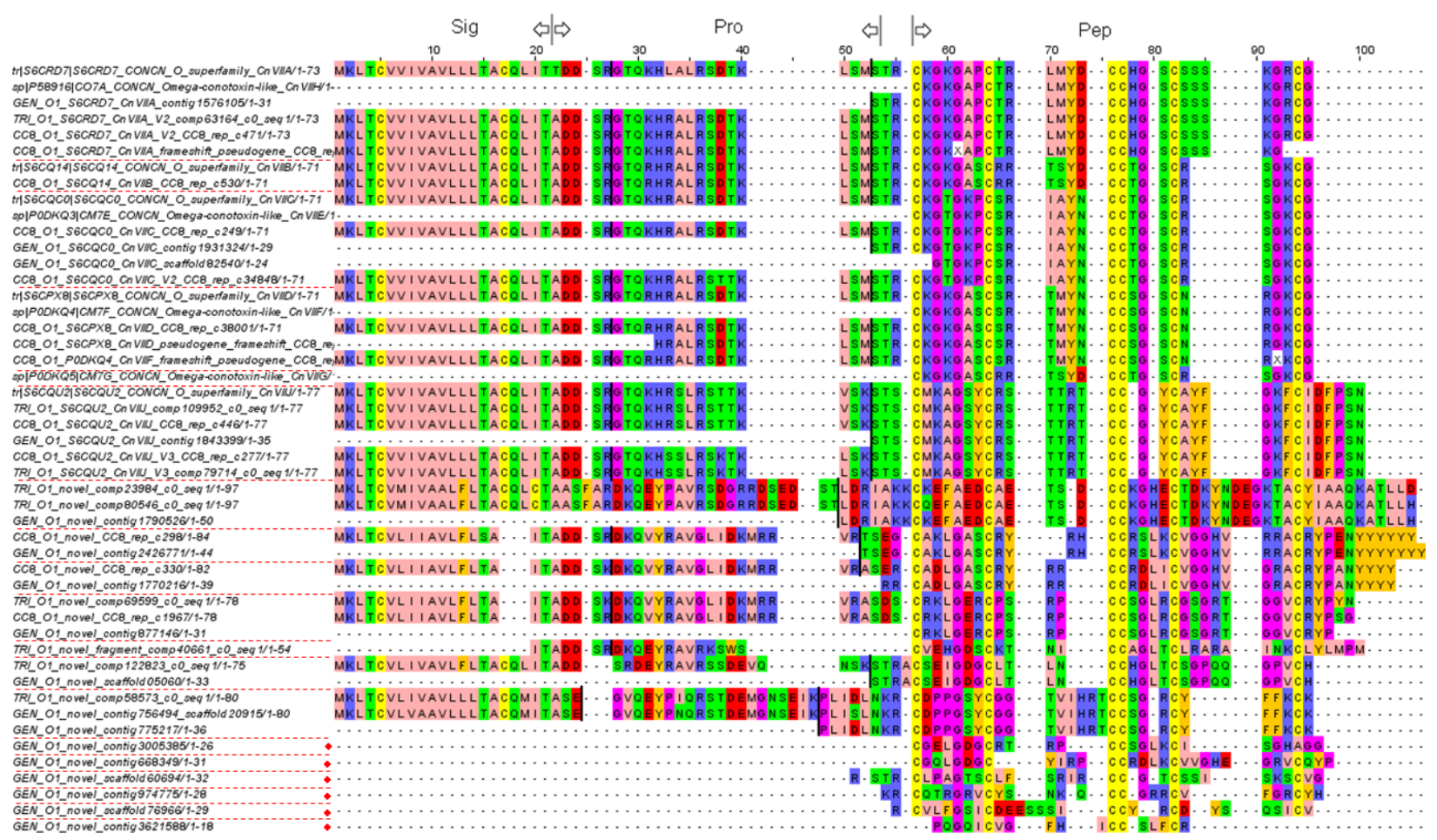

264 Figure 4. Example of conopeptide gene clusters. A subset of O1 superfamily gene clusters is

265 shown. Red lines denote boundaries of gene clusters. Red dots indicate "dubious" genes, which

266 show some similarity with conopeptides, but are not counted as conopeptide genes. 
Table 1. Number of conopeptide genes predicted from the $C$. consors genome and transcriptomes, ordered by superfamilies.

\begin{tabular}{|c|c|c|c|c|}
\hline $\begin{array}{l}\text { Super- } \\
\text { Family }\end{array}$ & Alignment & $\begin{array}{c}\text { UniProt } \\
\text { genes } \\
\text { (present } \\
\text { in our } \\
\text { datasets) }\end{array}$ & $\begin{array}{l}\text { Novel } \\
\text { genes }\end{array}$ & $\begin{array}{c}\text { Dubious } \\
\text { genes }\end{array}$ \\
\hline
\end{tabular}

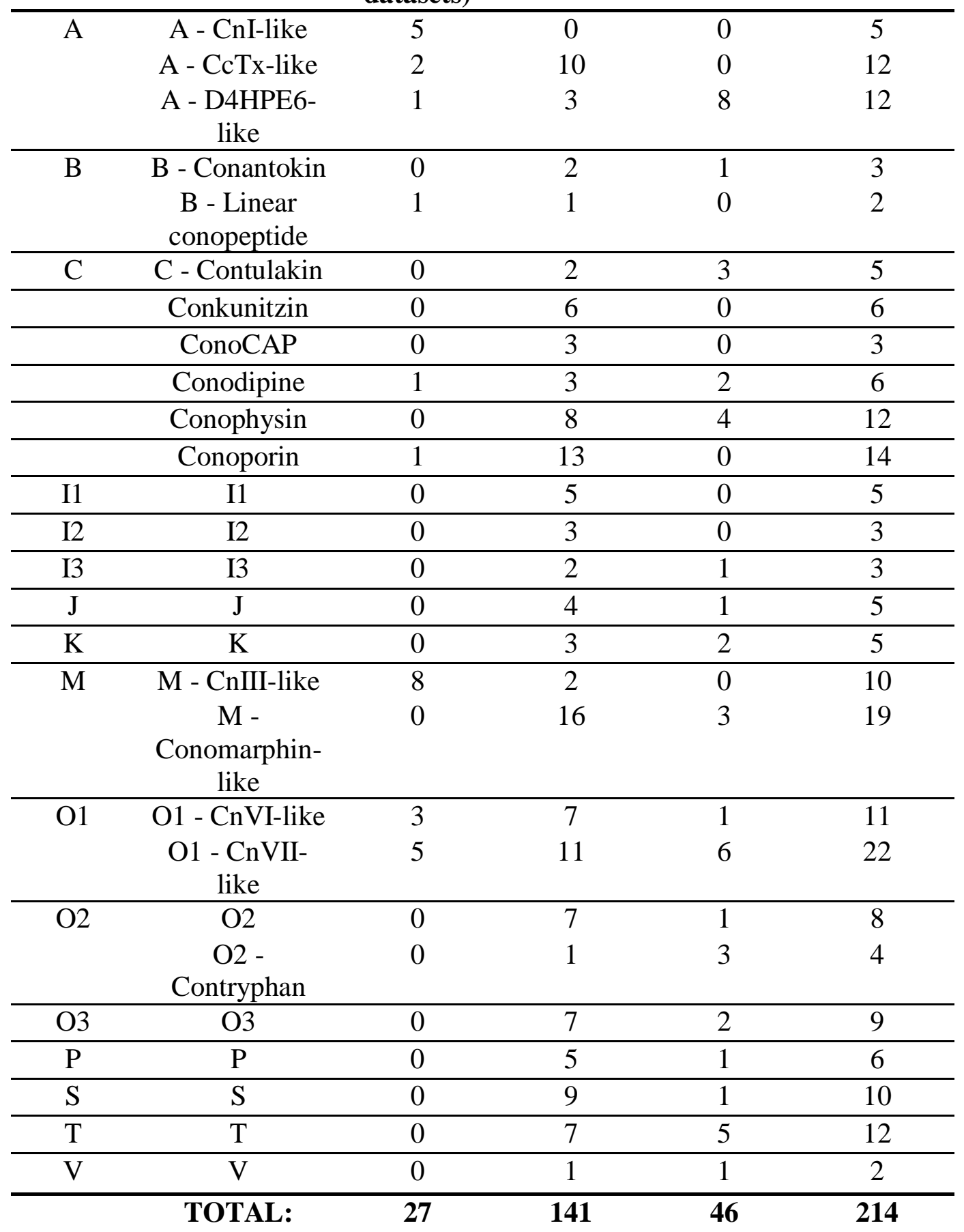




\section{Conopeptide genes in genome}

272 The majority of conopeptide superfamilies are known to contain introns that separate different

273 functional domains (Olivera et al., 1999). The genome sequence allows us to identify the

274 genomic structure of some conopeptide genes. Sequences that code for signal, propeptide, and

275 mature peptide domains were retrieved for 15 conopeptides from 14 superfamilies (Figure 5). It

276 is noteworthy that we can identify several different exon-intron organizations within the

277 conopeptide genes. The first exon of the most abundant type encodes for the complete signal

278 peptide sequence together with a variable length fragment of a pro-peptide, while the first exon

279 of genes encoding type A, I1, I3, and M conopeptides encode the entire signal sequence. Pro-

280 peptides appear to be encoded by one, two, or three different exons. Only conodipine genes are

281 devoid of pro-peptide sequences. Finally, in the unique case of J-conopeptides, their genes

282 appear to be made of a unique encoding exon containing, successively, a signal, an N-terminus

283 pro- and a mature peptide, followed by a C-terminus pro-sequence. 


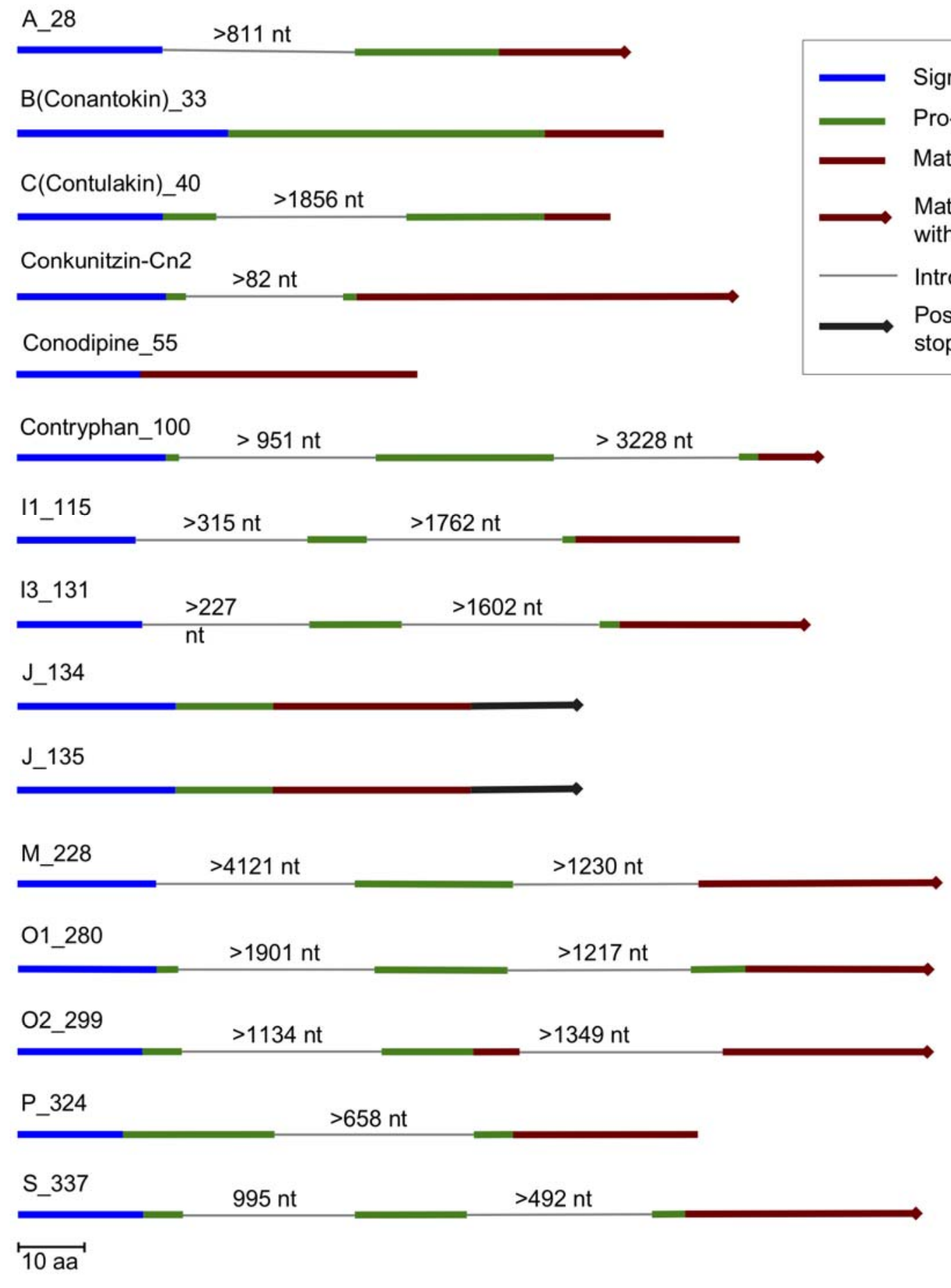

285 Figure 5. Conopeptide gene structures within the genome of $C$. consors. Each sample

286 represents one conopeptide gene. The peptide coding sequences (CS) for signal, pro- and mature

287 peptides are represented by bold blue, green, and red lines. The length of each line is 
288 proportional to the number of amino acids. The introns are represented as thin grey lines and the

289 length of the intron sequences is indicated in nucleotides above each line. The symbol '>'

290 indicates that this gene was not assembled into a single contig and that the intron length is

291 therefore not precisely known. Sequences of the conopeptide genes and additional information

292 are available in Supplementary Data S3.

\section{Conclusions}

295 The annotation of a fish-hunting cone snail C. consors genome and transcriptome gives us a 296 closer opportunity to peek into the complexity of its genes. The analysis of the combined eight 297 different transcriptomic and genomic datasets resulted 17,715 full-length protein sequences. In 298 addition, 168 conopeptide sequences were identified and in several cases the gene structures of 299 conopeptide superfamilies were characterized. We have found several gene coding clusters that might represent novel conopeptides and are therefore good candidates for future studies.

\section{Supplemental information}

303 The following additional data are available with the online version of this paper. Supplemental

304 Article S1 contains a detailed description of all supplementary analysis and methods.

305 Supplemental Data S2 contains list of predicted RNA genes, clustered by RFam category.

306 Supplemental Data S3 contains alignments of conopeptides from each superfamily. Gene and

307 protein sequences predicted from transcriptome are included in Supplemental Data S4 as two 308 separate FASTA format files. 


\section{Authors' contributions}

MRe and RA were responsible for drafting the manuscript. MRo, LK, SL, TK, AB, VK, RA and

MRe analyzed the sequencing data. All authors have read and approved the final manuscript.

\section{Acknowledgements}

315

Tõnu Margus and Aleksander Sudakov gave advice on construction of phylogenetic trees and gene content analysis. We would like to thank Tim Stockwell, Philippe Favreau, Daniel Biass, Yves Terrat and Dusan Kordis for valuable discussions during the initial survey of the genome sequence data.

\section{Funding Statement}

AB, SL, RA, LK, MRo, ML, TK, VK and MRe were supported by the EU FP6 CONCO project, SF0180026s09 and IUT34-11 from the Estonian Ministry of Education and Research and by the EU ERDF grant No. 2014-2020.4.01.15-0012 (Estonian Center of Excellence in Genomics and Translational Medicine). RA was also supported by the EU ERDF grant No. 2014-2020.4.01.160125. The computational analysis was partly carried out on the High Performance Computing Center of University of Tartu.

\section{References}

Abdel-Rahman MA., Abdel-Nabi IM., El-Naggar MS., Abbas OA., Strong PN. 2011. Intraspecific variation in the venom of the vermivorous cone snail Conus vexillum. Comparative biochemistry and physiology. Toxicology \& pharmacology $\square:$ CBP 154:318- 
25. DOI: 10.1016/j.cbpc.2011.06.019.

Adema CM., Hillier LW., Jones CS., Loker ES., Knight M., Minx P., Oliveira G., Raghavan N., Shedlock A., do Amaral LR., Arican-Goktas HD., Assis JG., Baba EH., Baron OL., Bayne CJ., Bickham-Wright U., Biggar KK., Blouin M., Bonning BC., Botka C., Bridger JM., Buckley KM., Buddenborg SK., Lima Caldeira R., Carleton J., Carvalho OS., Castillo MG., Chalmers IW., Christensens M., Clifton S., Cosseau C., Coustau C., Cripps RM., CuestaAstroz Y., Cummins SF., di Stephano L., Dinguirard N., Duval D., Emrich S., Feschotte C., Feyereisen R., FitzGerald P., Fronick C., Fulton L., Galinier R., Gava SG., Geusz M., Geyer KK., Giraldo-Calderón GI., de Souza Gomes M., Gordy MA., Gourbal B., Grunau C., Hanington PC., Hoffmann KF., Hughes D., Humphries J., Jackson DJ., Jannotti-Passos LK., de Jesus Jeremias W., Jobling S., Kamel B., Kapusta A., Kaur S., Koene JM., Kohn AB., Lawson D., Lawton SP., Liang D., Limpanont Y., Liu S., Lockyer AE., Lovato TL., Ludolf F., Magrini V., McManus DP., Medina M., Misra M., Mitta G., Mkoji GM., Montague MJ., Montelongo C., Moroz LL., Munoz-Torres MC., Niazi U., Noble LR., Oliveira FS., Pais FS., Papenfuss AT., Peace R., Pena JJ., Pila EA., Quelais T., Raney BJ., Rast JP., Rollinson D., Rosse IC., Rotgans B., Routledge EJ., Ryan KM., Scholte LLS., Storey KB., Swain M., Tennessen JA., Tomlinson C., Trujillo DL., Volpi E V., Walker AJ., Wang T., Wannaporn I., Warren WC., Wu X-J., Yoshino TP., Yusuf M., Zhang S-M., Zhao M., Wilson RK. 2017. Whole genome analysis of a schistosomiasis-transmitting freshwater snail. Nature Communications 8:15451. DOI: 10.1038/ncomms 15451.

Albertin CB., Simakov O., Mitros T., Wang ZY., Pungor JR., Edsinger-Gonzales E., Brenner S., Ragsdale CW., Rokhsar DS. 2015. The octopus genome and the evolution of cephalopod neural and morphological novelties. Nature 524:220-224. DOI: 10.1038/nature14668.

Altschul SF., Madden TL., Schäffer AA., Zhang J., Zhang Z., Miller W., Lipman DJ. 1997. Gapped BLAST and PSI-BLAST: a new generation of protein database search programs. Nucleic acids research 25:3389-402.

Biass D., Dutertre S., Gerbault A., Menou J-L., Offord R., Favreau P., Stöcklin R. 2009. Comparative proteomic study of the venom of the piscivorous cone snail Conus consors. Journal of proteomics 72:210-8. DOI: 10.1016/j.jprot.2009.01.019. 
Dutertre S., Biass D., Stöcklin R., Favreau P. 2010. Dramatic intraspecimen variations within the injected venom of Conus consors: an unsuspected contribution to venom diversity. Toxicon $\square$ : official journal of the International Society on Toxinology 55:1453-62. DOI: 10.1016/j.toxicon.2010.02.025.

Dutertre S., Jin A., Kaas Q., Jones A., Alewood PF., Lewis RJ. 2013. Deep venomics reveals the mechanism for expanded peptide diversity in cone snail venom. Molecular \& cellular proteomics $\square:$ MCP 12:312-29. DOI: 10.1074/mcp.M112.021469.

Dutertre S., Jin A-H., Vetter I., Hamilton B., Sunagar K., Lavergne V., Dutertre V., Fry BG., Antunes A., Venter DJ., Alewood PF., Lewis RJ. 2014. Evolution of separate predationand defence-evoked venoms in carnivorous cone snails. Nature communications 5:3521. DOI: $10.1038 /$ ncomms 4521 .

Eddy SR. 2011. Accelerated Profile HMM Searches. PLoS computational biology 7:e1002195. DOI: 10.1371/journal.pcbi.1002195.

Favreau P., Stöcklin R. 2009. Marine snail venoms: use and trends in receptor and channel neuropharmacology. Current opinion in pharmacology 9:594-601. DOI: 10.1016/j.coph.2009.05.006.

Fu Y., Li C., Dong S., Wu Y., Zhangsun D., Luo S. 2018. Discovery Methodology of Novel Conotoxins from Conus Species. Marine Drugs 16:417. DOI: 10.3390/md16110417.

Gao B., Peng C., Yang J., Yi Y., Zhang J., Shi Q. 2017. Cone Snails: A Big Store of Conotoxins for Novel Drug Discovery. Toxins 9:397. DOI: 10.3390/toxins9120397.

Grabherr MG., Haas BJ., Yassour M., Levin JZ., Thompson DA., Amit I., Adiconis X., Fan L., Raychowdhury R., Zeng Q., Chen Z., Mauceli E., Hacohen N., Gnirke A., Rhind N., di Palma F., Birren BW., Nusbaum C., Lindblad-Toh K., Friedman N., Regev A. 2011. Fulllength transcriptome assembly from RNA-Seq data without a reference genome. Nature biotechnology 29:644-52. DOI: 10.1038/nbt.1883.

Han TS., Teichert RW., Olivera BM., Bulaj G. 2008. Conus venoms - a rich source of peptidebased therapeutics. Current pharmaceutical design 14:2462-79. 
Hedges SB., Marin J., Suleski M., Paymer M., Kumar S. 2015. Tree of life reveals clock-like speciation and diversification. Molecular Biology and Evolution:msv037-. DOI: 10.1093/molbev/msv037.

Kocot KM., Cannon JT., Todt C., Citarella MR., Kohn AB., Meyer A., Santos SR., Schander C., Moroz LL., Lieb B., Halanych KM. 2011. Phylogenomics reveals deep molluscan relationships. Nature 477:452-6. DOI: 10.1038/nature10382.

Laht S., Koua D., Kaplinski L., Lisacek F., Stöcklin R., Remm M. 2012. Identification and classification of conopeptides using profile Hidden Markov Models. Biochimica et biophysica acta 1824:488-92. DOI: 10.1016/j.bbapap.2011.12.004.

Lavergne V., Harliwong I., Jones A., Miller D., Taft RJ., Alewood PF. 2015. Optimized deeptargeted proteotranscriptomic profiling reveals unexplored Conus toxin diversity and novel cysteine frameworks. Proceedings of the National Academy of Sciences 112:E3782-E3791. DOI: $10.1073 /$ pnas.1501334112.

Lewis RJ., Dutertre S., Vetter I., Christie MJ. 2012. Conus Venom Peptide Pharmacology. Pharmacological Reviews 64:259-298. DOI: 10.1124/pr.111.005322.

Li W., Godzik A. 2006. Cd-hit: a fast program for clustering and comparing large sets of protein or nucleotide sequences. Bioinformatics (Oxford, England) 22:1658-9. DOI: 10.1093/bioinformatics/btl158.

Liu Z., Bartels P., Sadeghi M., Du T., Dai Q., Zhu C., Yu S., Wang S., Dong M., Sun T., Guo J., Peng S., Jiang L., Adams DJ., Dai Q. 2018. A novel $\alpha$-conopeptide Eu1.6 inhibits N-type (CaV2.2) calcium channels and exhibits potent analgesic activity. Scientific Reports 8:1004. DOI: 10.1038/s41598-017-18479-4.

Lowe TM., Eddy SR. 1997. tRNAscan-SE: a program for improved detection of transfer RNA genes in genomic sequence. Nucleic acids research 25:955-64.

Luo R., Liu B., Xie Y., Li Z., Huang W., Yuan J., He G., Chen Y., Pan Q., Liu Y., Tang J., Wu G., Zhang H., Shi Y., Liu Y., Yu C., Wang B., Lu Y., Han C., Cheung DW., Yiu S-M., Peng S., Xiaoqian Z., Liu G., Liao X., Li Y., Yang H., Wang J., Lam T-W., Wang J. 2012. 

SOAPdenovo2: an empirically improved memory-efficient short-read de novo assembler. GigaScience 1:18. DOI: 10.1186/2047-217X-1-18.

Mir R., Karim S., Amjad Kamal M., M. Wilson C., Mirza Z. 2016. Conotoxins: Structure, Therapeutic Potential and Pharmacological Applications. Current Pharmaceutical Design 22:582-589. DOI: 10.2174/1381612822666151124234715.

Nawrocki EP., Kolbe DL., Eddy SR. 2009. Infernal 1.0: inference of RNA alignments. Bioinformatics (Oxford, England) 25:1335-7. DOI: 10.1093/bioinformatics/btp157.

Neves JLB., Lin Z., Imperial JS., Antunes A., Vasconcelos V., Olivera BM., Schmidt EW. 2015. Small Molecules in the Cone Snail Arsenal. Organic Letters 17:4933-4935. DOI: 10.1021/acs.orglett.5b02389.

Olivera BM. 1997. E.E. Just Lecture, 1996. Conus venom peptides, receptor and ion channel targets, and drug design: 50 million years of neuropharmacology. Molecular biology of the cell 8:2101-9.

Olivera BM., Walker C., Cartier GE., Hooper D., Santos AD., Schoenfeld R., Shetty R., Watkins M., Bandyopadhyay P., Hillyard DR. 1999. Speciation of cone snails and interspecific hyperdivergence of their venom peptides. Potential evolutionary significance of introns. Annals of the New York Academy of Sciences 870:223-37.

Parra G., Bradnam K., Korf I. 2007. CEGMA: a pipeline to accurately annotate core genes in eukaryotic genomes. Bioinformatics (Oxford, England) 23:1061-7. DOI: 10.1093/bioinformatics/btm071.

Parra G., Bradnam K., Ning Z., Keane T., Korf I. 2009. Assessing the gene space in draft genomes. Nucleic acids research 37:289-97. DOI: 10.1093/nar/gkn916.

Puillandre N., Bouchet P., Duda TF., Kauferstein S., Kohn AJ., Olivera BM., Watkins M., Meyer C. 2014. Molecular phylogeny and evolution of the cone snails (Gastropoda, Conoidea). Molecular Phylogenetics and Evolution 78:290-303. DOI: 10.1016/j.ympev.2014.05.023.

Puillandre N., Koua D., Favreau P., Olivera BM., Stöcklin R. 2012. Molecular phylogeny, classification and evolution of conopeptides. Journal of molecular evolution 74:297-309. 
DOI: $10.1007 / \mathrm{s} 00239-012-9507-2$.

Rice P., Longden I., Bleasby A. 2000. EMBOSS: the European Molecular Biology Open Software Suite. Trends in genetics $\square:$ TIG 16:276-7.

Schmieder R., Edwards R. 2011. Fast identification and removal of sequence contamination from genomic and metagenomic datasets. PloS one 6:e17288. DOI: 10.1371/journal.pone.0017288.

Simakov O., Marletaz F., Cho S-J., Edsinger-Gonzales E., Havlak P., Hellsten U., Kuo D-H., Larsson T., Lv J., Arendt D., Savage R., Osoegawa K., de Jong P., Grimwood J., Chapman JA., Shapiro H., Aerts A., Otillar RP., Terry AY., Boore JL., Grigoriev I V., Lindberg DR., Seaver EC., Weisblat DA., Putnam NH., Rokhsar DS. 2013. Insights into bilaterian evolution from three spiralian genomes. Nature 493:526-31. DOI: 10.1038/nature11696.

Smith CR., Smith CD., Robertson HM., Helmkampf M., Zimin A., Yandell M., Holt C., Hu H., Abouheif E., Benton R., Cash E., Croset V., Currie CR., Elhaik E., Elsik CG., Favé M-J., Fernandes V., Gibson JD., Graur D., Gronenberg W., Grubbs KJ., Hagen DE., Viniegra ASI., Johnson BR., Johnson RM., Khila A., Kim JW., Mathis KA., Munoz-Torres MC., Murphy MC., Mustard JA., Nakamura R., Niehuis O., Nigam S., Overson RP., Placek JE., Rajakumar R., Reese JT., Suen G., Tao S., Torres CW., Tsutsui ND., Viljakainen L., Wolschin F., Gadau J. 2011a. Draft genome of the red harvester ant Pogonomyrmex barbatus. Proceedings of the National Academy of Sciences of the United States of America 108:5667-72. DOI: 10.1073/pnas.1007901108.

Smith SA., Wilson NG., Goetz FE., Feehery C., Andrade SCS., Rouse GW., Giribet G., Dunn CW. 2011b. Resolving the evolutionary relationships of molluscs with phylogenomic tools. Nature 480:364-367. DOI: 10.1038/nature10526.

Suzek BE., Huang H., McGarvey P., Mazumder R., Wu CH. 2007. UniRef: comprehensive and non-redundant UniProt reference clusters. Bioinformatics (Oxford, England) 23:1282-8. DOI: 10.1093/bioinformatics/btm098.

Takeuchi T., Kawashima T., Koyanagi R., Gyoja F., Tanaka M., Ikuta T., Shoguchi E., Fujiwara M., Shinzato C., Hisata K., Fujie M., Usami T., Nagai K., Maeyama K., Okamoto K., Aoki 

H., Ishikawa T., Masaoka T., Fujiwara A., Endo K., Endo H., Nagasawa H., Kinoshita S., Asakawa S., Watabe S., Satoh N. 2012. Draft Genome of the Pearl Oyster Pinctada fucata: A Platform for Understanding Bivalve Biology. DNA Research 19:117-130. DOI: 10.1093/dnares/dss005.

Terrat Y., Biass D., Dutertre S., Favreau P., Remm M., Stöcklin R., Piquemal D., Ducancel F. 2012. High-resolution picture of a venom gland transcriptome: case study with the marine snail Conus consors. Toxicon $\square:$ official journal of the International Society on Toxinology 59:34-46. DOI: 10.1016/j.toxicon.2011.10.001.

The UniProt Consortium. 2015. UniProt: a hub for protein information. Nucleic Acids Research 43:D204-212. DOI: 10.1093/nar/gku989.

Violette A., Biass D., Dutertre S., Koua D., Piquemal D., Pierrat F., Stöcklin R., Favreau P. 2012. Large-scale discovery of conopeptides and conoproteins in the injectable venom of a fish-hunting cone snail using a combined proteomic and transcriptomic approach. Journal of proteomics 75:5215-25. DOI: 10.1016/j.jprot.2012.06.001.

Wurm Y., Wang J., Riba-Grognuz O., Corona M., Nygaard S., Hunt BG., Ingram KK., Falquet L., Nipitwattanaphon M., Gotzek D., Dijkstra MB., Oettler J., Comtesse F., Shih C-J., Wu W-J., Yang C-C., Thomas J., Beaudoing E., Pradervand S., Flegel V., Cook ED., Fabbretti R., Stockinger H., Long L., Farmerie WG., Oakey J., Boomsma JJ., Pamilo P., Yi S V., Heinze J., Goodisman MAD., Farinelli L., Harshman K., Hulo N., Cerutti L., Xenarios I., Shoemaker D., Keller L. 2011. The genome of the fire ant Solenopsis invicta. Proceedings of the National Academy of Sciences of the United States of America 108:5679-84. DOI: 10.1073/pnas.1009690108.

Zhang G., Fang X., Guo X., Li L., Luo R., Xu F., Yang P., Zhang L., Wang X., Qi H., Xiong Z., Que H., Xie Y., Holland PWH., Paps J., Zhu Y., Wu F., Chen Y., Wang J., Peng C., Meng J., Yang L., Liu J., Wen B., Zhang N., Huang Z., Zhu Q., Feng Y., Mount A., Hedgecock D., Xu Z., Liu Y., Domazet-Lošo T., Du Y., Sun X., Zhang S., Liu B., Cheng P., Jiang X., Li J., Fan D., Wang W., Fu W., Wang T., Wang B., Zhang J., Peng Z., Li Y., Li N., Wang J., Chen M., He Y., Tan F., Song X., Zheng Q., Huang R., Yang H., Du X., Chen L., Yang M., Gaffney PM., Wang S., Luo L., She Z., Ming Y., Huang W., Zhang S., Huang B., 
